\title{
Dynamic characterization of Galfenol
}

\author{
Justin J. Scheidler $^{a}$, Vivake M. Asnani ${ }^{b}$, Zhangxian Deng $^{a}$, Marcelo J. Dapino ${ }^{a}$ \\ ${ }^{a}$ NSF I/UCRC on Smart Vehicle Concepts, Department of Mechanical and Aerospace \\ Engineering, The Ohio State University, Columbus, OH, USA, 43210 \\ ${ }^{b}$ Rotating and Drive Systems Branch, Materials and Structures Division, NASA Glenn \\ Research Center, Cleveland, OH 44135
}

\begin{abstract}
A novel and precise characterization of the constitutive behavior of solid and laminated research-grade, polycrystalline Galfenol $\left(\mathrm{Fe}_{81.6} \mathrm{Ga}_{18.4}\right)$ under under quasi-static $(1 \mathrm{~Hz})$ and dynamic $(4$ to $1000 \mathrm{~Hz})$ stress loadings was recently conducted by the authors. This paper summarizes the characterization by focusing on the experimental design and the dynamic sensing response of the solid Galfenol specimen. Mechanical loads are applied using a high frequency load frame. The dynamic stress amplitude for minor and major loops is 2.88 and $31.4 \mathrm{MPa}$, respectively. Dynamic minor and major loops are measured for the bias condition resulting in maximum, quasi-static sensitivity. Three key sources of error in the dynamic measurements are accounted for: (1) electromagnetic noise in strain signals due to Galfenol's magnetic response, (2) error in load signals due to the inertial force of fixturing, and (3) time delays imposed by conditioning electronics. For dynamic characterization, strain error is kept below $1.2 \%$ of full scale by wiring two collocated gauges in series (noise cancellation) and through lead wire weaving. Inertial force error is kept below $0.41 \%$ by measuring the dynamic force in the specimen using a nearly collocated piezoelectric load washer. The phase response of all conditioning electronics is explicitly measured and corrected for. In general, as frequency increases, the sensing response becomes more linear due to an increase in eddy currents. The location of positive and negative saturation is the same at all frequencies. As frequency increases above about 100 $\mathrm{Hz}$, the elbow in the strain versus stress response disappears as the active (soft) regime stiffens toward the passive (hard) regime.
\end{abstract}

Keywords: Galfenol, magnetomechanical characterization, dynamic stress, dynamic sensing

\section{INTRODUCTION}

Magnetostrictive materials, such as Galfenol $\left(\mathrm{Fe}_{1-x} \mathrm{Ga}_{x}, 0.13 \leq x \leq 0.29\right)$, can transduce energy between magnetic and mechanical domains, thereby providing actuation, sensing, and secondary effects. Galfenol is a particularly useful type of magnetostrictive material, because it has high tensile strength $(\approx 500 \mathrm{MPa})$, very low magnetomechanical hysteresis, high magnetic permeability, and fairly constant active properties in the temperature range -20 to $80^{\circ} \mathrm{C} .{ }^{1}$ Through its unique combination of active properties and mechanical strength, Galfenol has the potential to transform the smart materials industry by allowing for the development of active, load-bearing devices, such as sensors, energy harvesters, vibration dampers, and variable stiffness components. One of the primary technical challenges is the very limited experimental data on Galfenol's frequency-dependent response to dynamic stress, which is critically important for the design of such devices. Galfenol's magnetic response to small amplitude dynamic stress (up to $2.8 \mathrm{MPa}, 1 \mathrm{kHz}$ and 6.5 $\mathrm{MPa}, 10 \mathrm{~Hz}$, respectively) has been reported $;^{2,3}$ however, the phase response of the measurement systems was neglected, which significantly increases the uncertainty in hysteresis measurements. No other measurements have been published.

Further author information: (Send correspondence to M.J.D)

J.J.S.: E-mail: scheidler.8@osu.edu, Telephone: 1-614-247-7480

M.J.D.: E-mail: dapino.1@osu.edu, Telephone: 1-614-688-3689

Behavior and Mechanics of Multifunctional Materials and Composites 2015, edited by Nakhiah C. Goulbourne Proc. of SPIE Vol. 9432, 94320J · @ 2015 SPIE · CCC code: 0277-786X/15/\$18 · doi: 10.1117/12.2085573

Proc. of SPIE Vol. 9432 94320J-1 
This paper summarizes the authors' recent report, ${ }^{4}$ in which a detailed and precise characterization of Galfenol's $\left(\mathrm{Fe}_{81.6} \mathrm{Ga}_{18.4}\right)$ magnetic and mechanical responses to dynamic compressive stresses up to $31 \mathrm{MPa}$ and $1 \mathrm{kHz}$ was presented. The objective was to measure the 1D, dynamic sensing response of the material and to quantify from the response, the frequency dependence of the material properties for 1D sensing. This was accomplished by controlling the axial, dynamic stress and static magnetic field over a specific region of a Galfenol rod, and measuring the axial strain and magnetic flux density. The complete report considered the quasi-static and dynamic response of solid and laminated Galfenol rods, whereas this work focuses on the dynamic sensing response of the solid rod. First, the design of the experiment is discussed, including specimen design, uncertainty analysis and calibration methods, experimental setup and methods, and data processing methods. Next, the mechanical and magnetic responses of the solid rod under two magnetic bias conditions, constant current and constant magnetic field, is given, along with a discussion of the observed trends.

\section{EXPERIMENTAL DESIGN \& SETUP}

\subsection{Specimen Design}

To allow for the use of the existing electromagnetic transducer (magnetic circuit) shown in Fig. 1a, a cylindrical specimen of diameter 0.25 in was used. This magnetic circuit generated a uniform, axially-oriented magnetic state in the gauge region of the specimen (Fig. 1b), while allowing for independent mechanical excitation. The magnetic circuit was successfully used in previous work by the authors. ${ }^{5,6}$

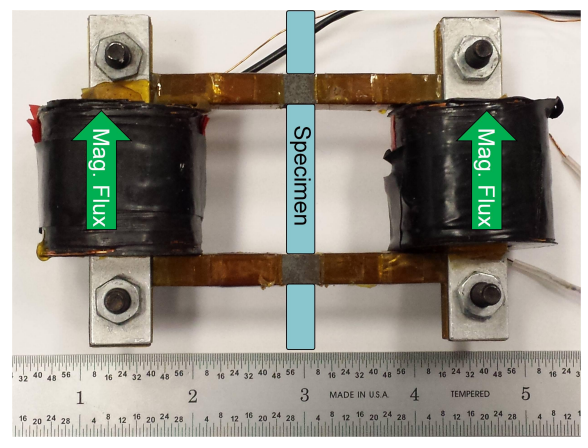

(a)

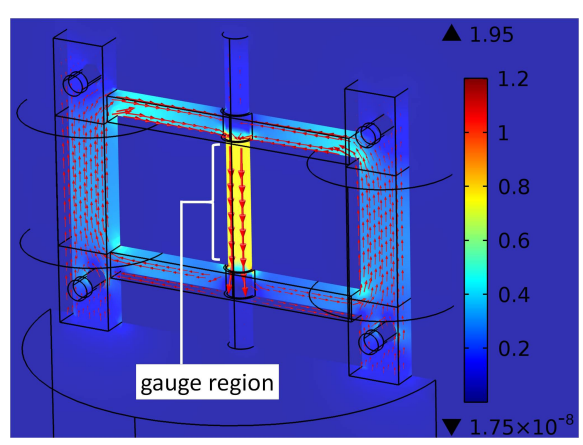

(b)

Figure 1: (a) magnetic circuit used to generate the magnetic state and (b) magnetostatic FE simulation of its magnetic response - magnetic flux density (arrows) and a slice plot of its norm (units of T).

The geometry of the specimen is depicted in Fig. 2. To assist with specimen design, particularly with tolerancing, relevant ASTM standards were reviewed, and specimen specifications were recorded. The specimen's manufacturer attempted to meet the most stringent ASTM-recommended tolerances, but could not do so for every specification. Due to the specimen's high length-to-diameter ratio, buckling of the rod under dynamic, axial compression was investigated. Using the minimum elastic modulus of the rod, $35 \mathrm{GPa}$, the 1st transverse bending natural frequency of the rod was approximated as a function of compressive stress. For the stresses used in this characterization, said natural frequency was about $1950 \mathrm{~Hz}$ or above. This value was considerably higher than the frequency limit of $1000 \mathrm{~Hz}$ and provided a good factor of safety from decreases in the natural frequency due to mass loading.

The specimen is a research-grade, $\langle 100\rangle$ oriented, polycrystalline rod grown using the free standing zone melt method at a rate of about $25 \mathrm{~mm} / \mathrm{hr}$. 


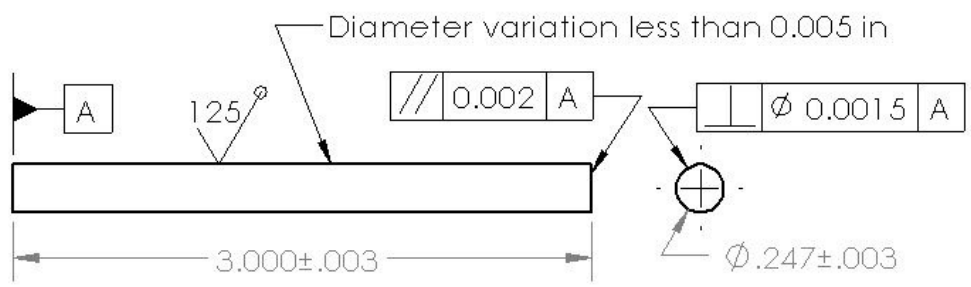

Figure 2: Dimensions and tolerances of the highly-textured, $\langle 100\rangle$ oriented, polycrystalline Galfenol $\left(\mathrm{Fe}_{81.6} \mathrm{Ga}_{18.4}\right)$ specimens; all specifications have units of in, except for the surface roughness, which is specified in units of $\mu \mathrm{in}$.

\subsection{Uncertainty Analysis \& Calibration Methods}

The uncertainty of each calculated quantity $u_{R}$ was quantified by the standard formula for the propagation of error,

$$
u_{R}= \pm\left[\sum_{i=1}^{n}\left(\frac{\partial R}{\partial x_{i}} u_{x_{i}}\right)^{2}\right]^{1 / 2}
$$

where $u_{x_{i}}$ is the uncertainty in the measured variable $x_{i}$ and $n$ denotes the number of variables on which the result $R$ depends. ${ }^{7}$ Individual uncertainties $u_{x_{i}}$ were calculated from the interpolation and instrument errors for each sensor, which were quantified from manufacturer-supplied specifications. ${ }^{4}$ For most sensors, the instrument error, and thus $u_{R}$, is a function of the magnitude of the measured quantity. This is illustrated in Fig. 3, which presents the uncertainty of the calculated strain and magnetic field. Table 1 summarizes the estimated range of uncertainty for each measurement and compares it to the suggested maximum uncertainty from ASTM standards. In most cases, the expected uncertainty approximately met ASTM recommendations. The magnetic flux density uncertainty exceeded its recommendation due to a high range-dependent uncertainty, which resulted from having a low signal-to-range ratio of $15 \%$.

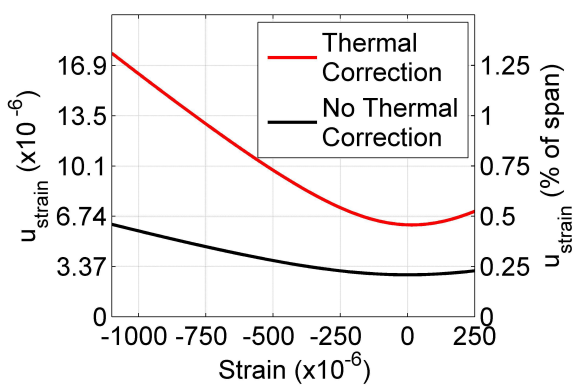

(a)

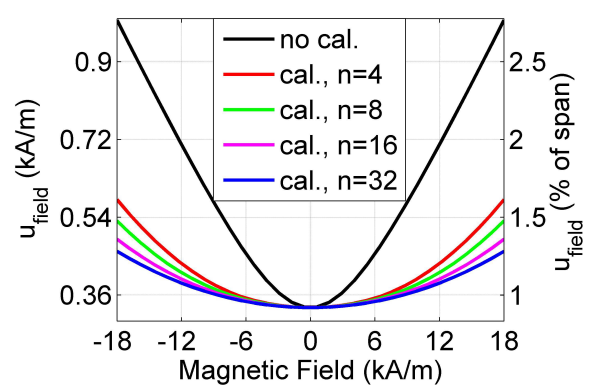

(b)

Figure 3: Reading-dependent uncertainty of (a) the strain, with and without correcting for thermal effects and (b) the magnetic field strength, with and without calibrating the Hall chip's sensitivity using a $n$-point calibration.

The method, experimental setup, and results for the calibration of each sensor and the linear power amplifier are detailed in the complete report. ${ }^{4}$ Most of the calibrations were conducted by comparing the device's output to an absolute reference or the output of a calibrated sensor. However, the strain gauges were shunt calibrated, whereas the magnetic flux density sensing (pick-up) coil was calibrated by measuring the magnetic response of a 0.25 in diameter $\mathrm{Ni} 200$ rod and comparing the resulting B-H loop to accurate measurements from the literature. ${ }^{8}$

The instruments used in the experiment are as follows. A Kepko BOP 72-6M linear amplifier, operated in current control mode, was selected based on its availability. Static force was measured using an Interface 
Table 1: Estimated uncertainty in the measured quantities.

\begin{tabular}{r|c|c}
\hline Physical quantity & $\begin{array}{c}\text { Measurement uncertainty, } \\
\pm \% \text { of span }\end{array}$ & $\begin{array}{c}\text { ASTM-suggested uncertainty } \\
\pm \% \text { of span }\end{array}$ \\
\hline Distance & 0.20 & $0.10-0.50$ \\
Area & 0.41 & 1.0 \\
Force & $0.065-1.0$ (static), $1.1-1.3$ (dynamic) & 1.0 \\
Stress & $0.065-1.1$ (static) $1.1-1.3$ (dynamic) & - \\
Temperature & $\pm 2.0^{\circ} \mathrm{C}$ & $\pm 1.0^{\circ} \mathrm{C}$ \\
Strain & $0.21-0.46$ & $0.10-1.0$ \\
Magnetic field strength & $0.92-1.3$ & 1.0 \\
Magnetic flux density & $1.4-1.9$ & 1.0 \\
\hline
\end{tabular}

1010ACK-500-B fatigue rated load cell and MTS 493.21 signal conditioner. As discussed in Section 2.3, dynamic forces vibrate the specimen and fixtures, resulting in inertial forces that cause error in measurements of the force applied to the specimen. To minimize this error, the dynamic force was measured with a Kistler 9001A piezoelectric load washer and Kistler 5010 charge amplifier, instead of the Interface load cell. A Type K thermocouple and Omega DRG-SC-TC signal conditioner were used to measure the temperature of the specimen. Strain measurements were obtained using Vishay Micro-Measurements EA-06-250BF-350/L strain gauges and a Vishay Micro-Measurements 2310 signal conditioner. An Allegro A1321LUA Hall chip was selected to measure the surface magnetic field, while a Lake Shore Model 480 fluxmeter and custom pick-up coil were used to measure the magnetic flux density.

\subsection{Experimental Setup \& Methods}

An MTS 831.50 dynamic load frame was used to excite the Galfenol specimen with quasi-static and dynamic axial forces up to $-2000 \mathrm{~N}$ and $1 \mathrm{kHz}$. Due to the high stiffness of the specimen, the load frame was operated in force control mode, instead of displacement control mode; this was realized using an MTS 793 FlexTest GT controller. The test setups used for quasi-static and dynamic testing are provided in Fig. 4a. Here, the thermocouple, Hall chip, sensing coil, and strain gauges can be seen installed on the Galfenol rod. Surrounding the rod is the magnetic circuit used to generate the magnetic field.

Any vibrating mass in-between the force transducer and specimen will generate inertial force that corrupts the force measurement. ASTM recommends that the inertial force error be less than $0.5 \%$ of the loading span. ${ }^{9}$ Using a two-degree-of-freedom lumped parameters model of the load path in the right image of Fig. $4 \mathrm{a}$, the inertial force error was approximated as

$$
\left|E_{2}\right|=\frac{1}{2}\left(1+\left|\frac{k_{2}\left(k_{3}-m_{3} \omega^{2}\right)}{m_{2} \omega^{2}\left(m_{3} \omega^{2}-\left(k_{2}+k_{3}\right)\right)}\right|\right)^{-1},
$$

where $m_{2}$ is the mass of the alignment puck, $\omega$ is the circular frequency of the excitation force, $k_{2}$ is the stiffness of the load washer, $m_{3}$ is the combined mass of the Al platen and magnetic circuit, and $k_{3}$ is the stiffness of the load cell. The magnitude of the error increases linearly with the mass in-between the specimen and force transducer (i.e., $m_{2}$ ). Eq. 2 is plotted in Fig. $4 \mathrm{~b}$ for the parameters of the actual test setup. Since the load washer is nearly collocated with the specimen, the inertial force error does not exceed the error threshold until $1077 \mathrm{~Hz}$.

Another key source of experimental error is the electromagnetic noise voltage induced in the strain gauges due to the time-varying magnetic response of the specimen. In the absence of noise reduction techniques, this error can exceed $200 \%$ of full scale for high frequency mechanical loading. As explained in the full report, strain error is kept below $1.2 \%$ of full scale by wiring two collocated gauges in series (noise cancellation) and through lead wire weaving. The complete report also details the wiring of the experiment, the magnetic field control, and the sensor reset method. ${ }^{4}$ 


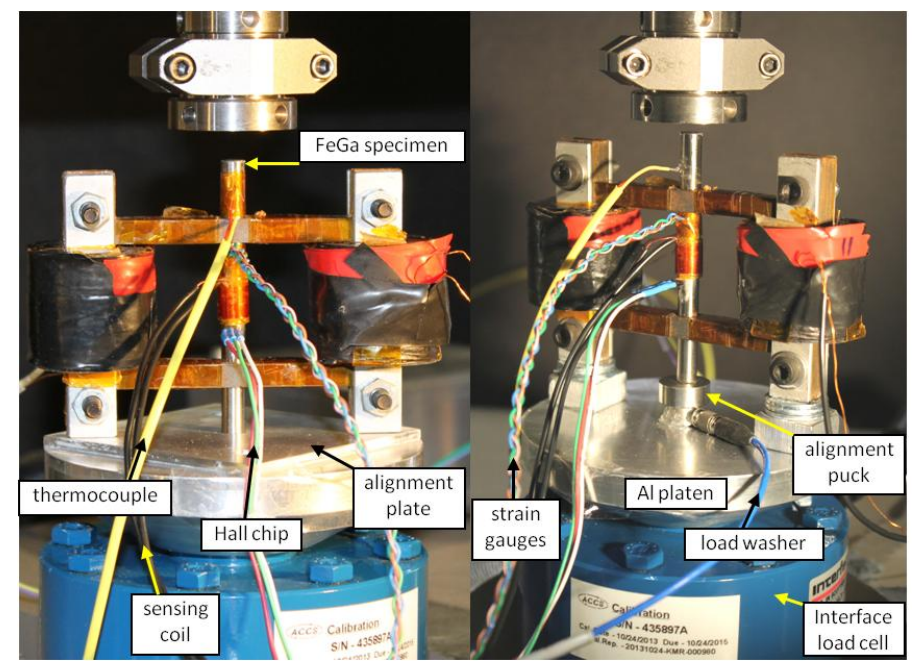

(a)

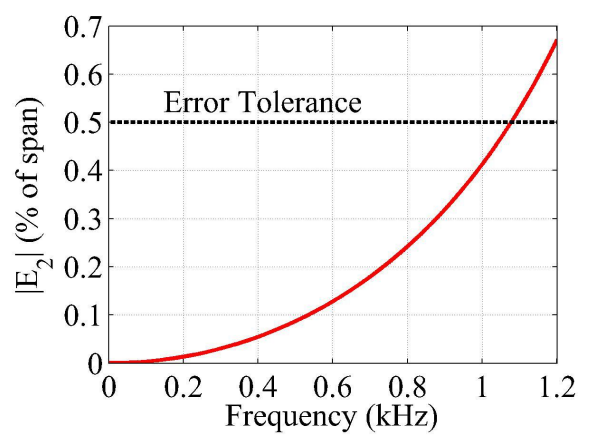

(b)

Figure 4: (a) experimental setup of the quasi-static (left) and dynamic (right) experiments in the MTS 831.50 dynamic load frame and (b) measurement error (\% of the loading span) in the force applied to the specimen due to the inertial force of fixturing.

\subsection{Data Processing Methods}

Each signal conditioner and corresponding anti-aliasing filter created a different phase shift, resulting in misalignment of the measured sensor signals. This misalignment created error in the size of the material characterization hysteresis loops. Typically, force and displacement signals are aligned by testing a sample that has near zero energy loss. ${ }^{10}$ This technique could not be applied for this system, as the mechanical and magnetic sensor signals needed to be mutually aligned. Instead, a new technique was used.

First, the sensors were removed and the sensor signals were simulated by manipulating a voltage signal produced by a function generator (Fig. 5). For the fluxmeter and the strain and load cell conditioners, generated signals were sent through voltage divider circuits to simulate the magnitude of each sensor's output while presenting each sensor's output impedance to the corresponding conditioner. An isolation amplifier was needed between the function generator and fluxmeter to prevent damage to the fluxmeter. The charge signal generated by the piezoelectric load washer was simulated by placing a capacitor in series with the function generator. The phase delay of each channel was measured with respect to the magnetic field sensor channel at multiple frequencies. A linear, least-squares regression was fit to each phase response. The phase delays were then corrected for in the frequency domain during post processing.

Further details of this unique phase alignment technique, in addition to a discussion of signal scaling, filtering, calculation of material properties, and evaluation of reversibility in the constitutive response, can be found in the complete report. ${ }^{4}$

\section{DYNAMIC SENSING MEASUREMENTS}

In this paper, only the dynamic sensing responses of the solid Galfenol rod are presented. The full report also details the quasi-static sensing and actuation responses of the solid and laminated Galfenol rods, the dynamic sensing responses of the laminated Galfenol rod, and the testing procedure. ${ }^{4}$

For the dynamic minor loops, the bias conditions were those for which the quasi-static magnetomechanical coupling of the solid rod was maximized (i.e., maximum sensitivity and minimum elastic modulus): $0.30 \mathrm{~A}$ and -9.93 MPa for constant current tests and $2.46 \mathrm{kA} / \mathrm{m}$ and $-7.96 \mathrm{MPa}$ for constant field tests. Dynamic major loops were measured for a mechanical bias of $-31.9 \mathrm{MPa}$ (roughly the midpoint of the stress range) and the same magnetic biases as used for dynamic minor loops. To determine the effect of forcing frequency 


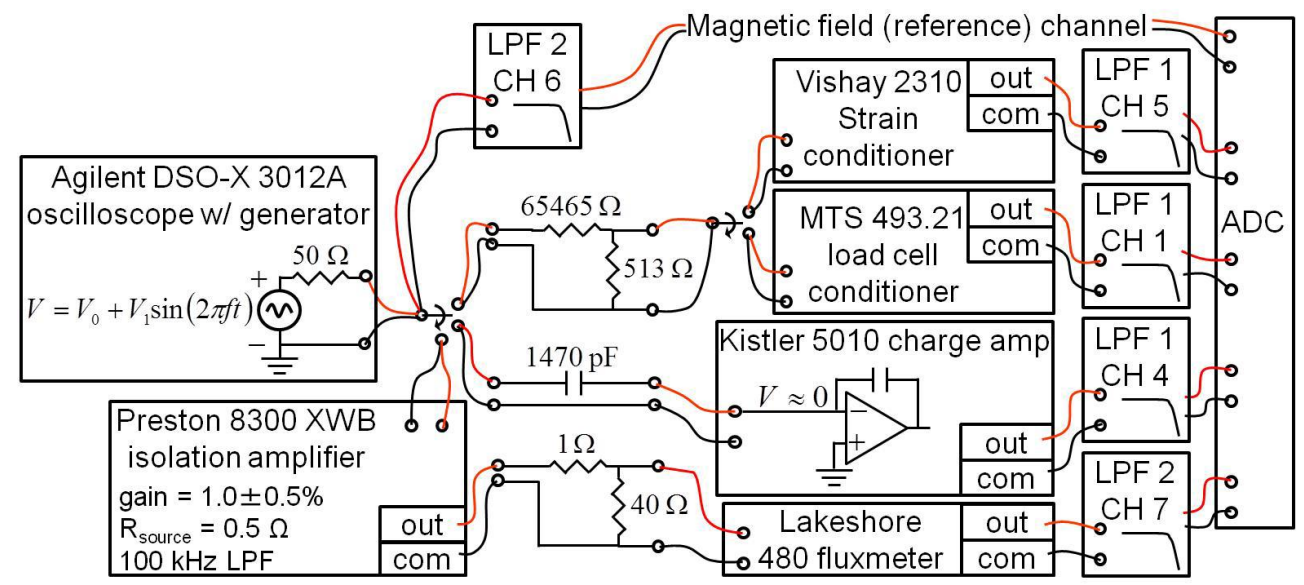

Figure 5: Experimental setup for measuring the phase response of the conditioning electronics for strain, load cell, load washer, and flux density signals.

on positive and negative saturation, dynamic major loops were also measured for a $0.80 \mathrm{~A}$ bias. The stress amplitude for major and minor loops was 2.88 and $31.4 \mathrm{MPa}$, respectively. Measurements are shown for forcing frequencies of 4, 10, 50, 100, 200, 400, 600, 800, and 1000 (or 975) Hz.

\subsection{Constant Current}

The major loop sensing responses of the solid rod under constant current are shown in Figs. 6a/6b and 6c/6d for bias currents of 0.30 and $0.80 \mathrm{~A}$, respectively. During each run, the bias current varies due to the power amplifier's imperfect current control. For both bias currents, current variation increases nearly linearly with frequency from $0.02 \mathrm{~mA}$ at $4 \mathrm{~Hz}$ to $14 \mathrm{~mA}$ at $1000 \mathrm{~Hz}$. The strain curves in Figs. 6a and 6c are successively shifted along the strain axis by $-110 \times 10^{-6}$ and $-125 \times 10^{-6}$, respectively, starting with the $10 \mathrm{~Hz}$ curve to more clearly visualize the behavior. At $4 \mathrm{~Hz}$, there is a distinct elbow in the strain response where the magnetostriction saturates and the elastic modulus reaches its saturated (stiff) value. For stresses greater than the elbow stress, magnetostriction occurs and the effective elastic modulus of the material is reduced. As frequency increases, the elbow disappears and the region of softened behavior stiffens until it coincides with the saturated, purely-elastic regime. Evident in the flux density versus stress plots in Figs. $6 \mathrm{~b}$ and $6 \mathrm{~d}$, hysteresis increases and sensitivity to stress decreases with frequency. These trends imply that at high frequency, magnetic moment rotation is severely inhibited in the solid Galfenol rod. As forcing frequency increases, the location of positive and negative saturation is unchanged.

Minor loop responses under constant current are depicted in Fig. 7. Current variation during each run is very small (less than $1.3 \mathrm{~mA}$ ) at all frequencies. Due to the low amplitude excitation and the bias condition, the response is nearly linear throughout the frequency range. An increase in hysteresis with frequency is clear in the strain versus stress and flux density versus stress plots. Similar to the major loop responses, but to a much lesser extent, the active behavior (i.e., flux density changes and a softened elastic modulus) is suppressed as frequency increases. Material properties calculated from the minor loops are given in the full report. ${ }^{4}$

\subsection{Constant Field}

Fig. 8 presents the dynamic, major loop sensing responses of the solid Galfenol rod at constant magnetic field. The maximum and standard deviation of the variation in field about the bias field increase monotonically with frequency from about 0.09 and $0.03 \mathrm{kA} / \mathrm{m}$ at $4 \mathrm{~Hz}$ to 0.8 and $0.4 \mathrm{kA} / \mathrm{m}$ at $1000 \mathrm{~Hz}$, respectively. For comparison, the change in magnetic field during major loop constant current tests was about $2.4 \mathrm{kA} / \mathrm{m}$ at low frequency and $2.1 \mathrm{kA} / \mathrm{m}$ at high frequency. Consequently, the magnetic field controller is only moderately effective above 100 to $200 \mathrm{~Hz}$. As expected, the active behavior of the rod under constant field conditions 


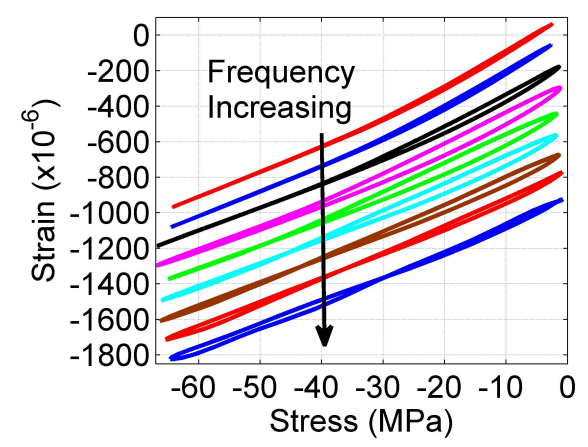

(a)

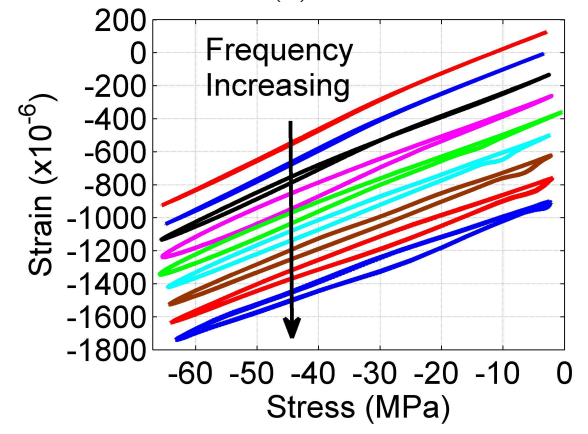

(c)

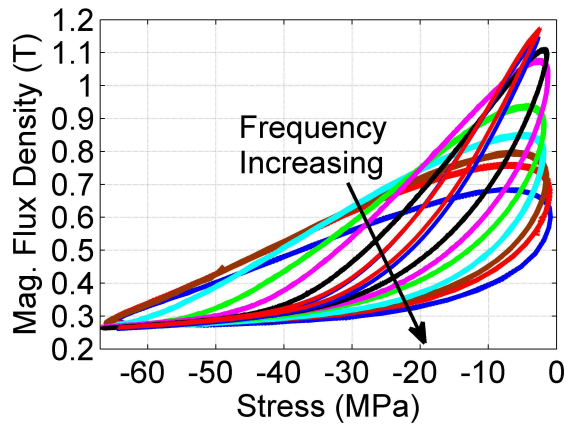

(b)

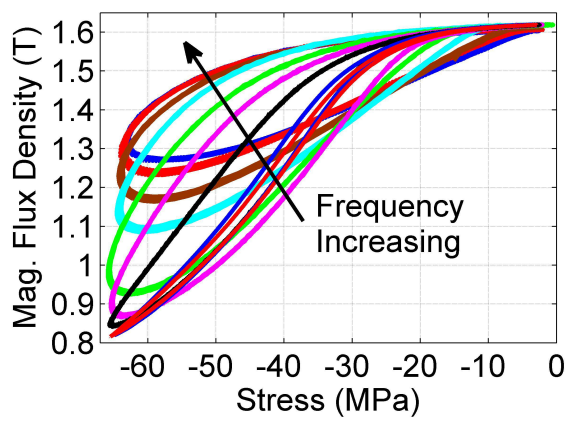

(d)

Figure 6: Major loop dynamic sensing responses of the solid Galfenol rod for bias currents of $0.3 \mathrm{~A}(\mathrm{a}, \mathrm{b})$ and $0.8 \mathrm{~A}(\mathrm{c}, \mathrm{d})$; strain responses for $0.3 \mathrm{~A}$ and $0.8 \mathrm{~A}$ biases are successively shifted by $-110 \times 10^{-6}$ and $-125 \times 10^{-6}$, respectively, for visualization purposes.

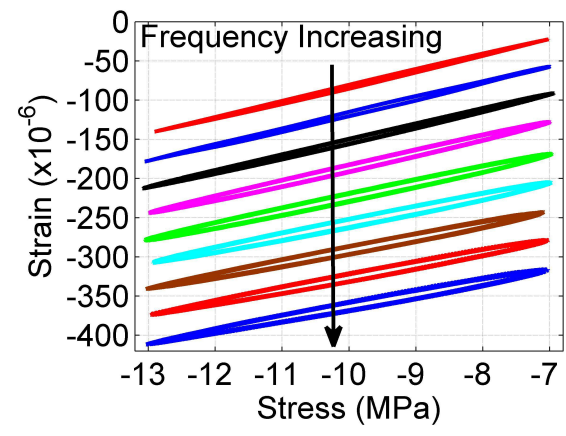

(a)

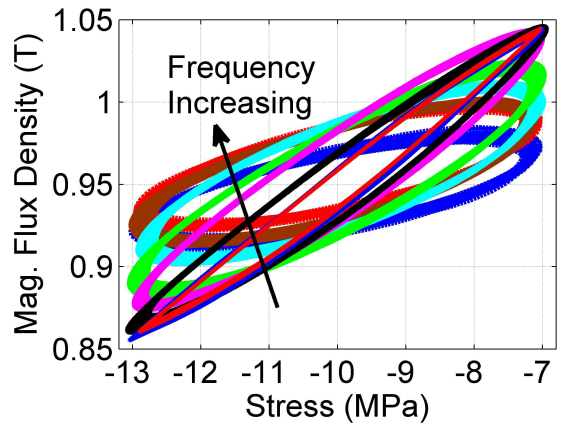

(b)

Figure 7: Minor loop dynamic sensing responses of the solid Galfenol rod for a bias current of $0.3 \mathrm{~A}$; strain responses are successively shifted by $-35 \times 10^{-6}$ for visualization purposes.

is steeper and has a larger amplitude than under constant current conditions. Trends in the strain and flux density curves mirror those in the constant current responses.

Fig. 9 shows the dynamic, minor loop response of the solid rod at constant field. The maximum variation in the magnetic field increases with frequency to a peak of about $0.23 \mathrm{kA} / \mathrm{m}$ at $600 \mathrm{~Hz}$, then decreases thereafter. Thus, the absolute variation in the magnetic field is considerably lower than for dynamic, major loop tests. However, the change in magnetic field during dynamic, minor loop constant current tests was only $0.9 \mathrm{kA} / \mathrm{m}$, compared to a change of $2.1 \mathrm{kA} / \mathrm{m}$ for major loops. Thus, the maximum variation in the controlled field is about $50 \%$ of the variation in the uncontrolled field. The slope of the flux density versus 


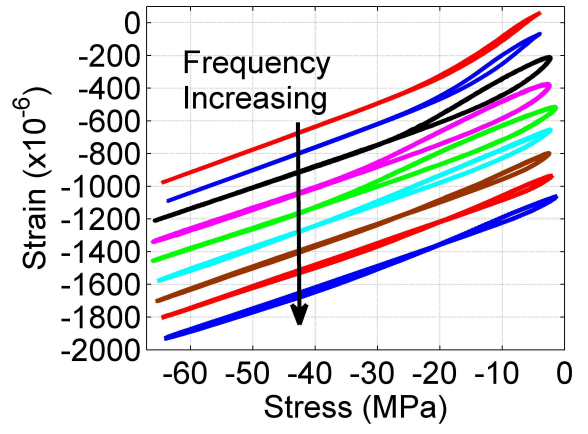

(a)

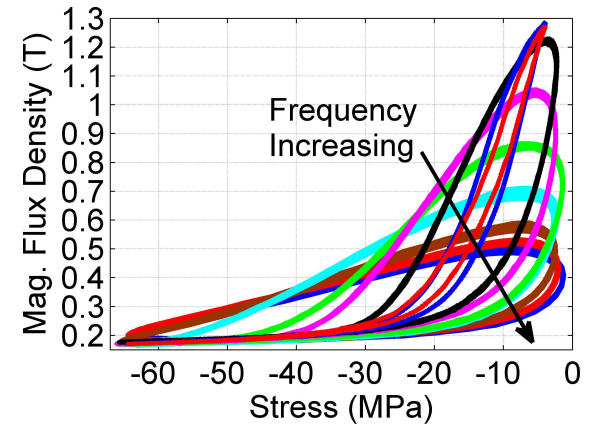

(b)

Figure 8: Major loop dynamic sensing responses of the solid Galfenol rod for a bias magnetic field of 2.46 $\mathrm{kA} / \mathrm{m}$; strain responses are successively shifted by $-125 \times 10^{-6}$ for visualization purposes.

stress and strain versus stress responses decreases with frequency faster than at constant current due the combined effects of suppressed magnetic moment rotation and degraded constant field control. The constant field minor loops exhibit considerably more hysteresis than the constant current minor loops up to $600 \mathrm{~Hz}$, but exhibit comparable hysteresis above $600 \mathrm{~Hz}$. This difference is quantified in the full report, ${ }^{4}$ where material properties are calculated from the minor loops.

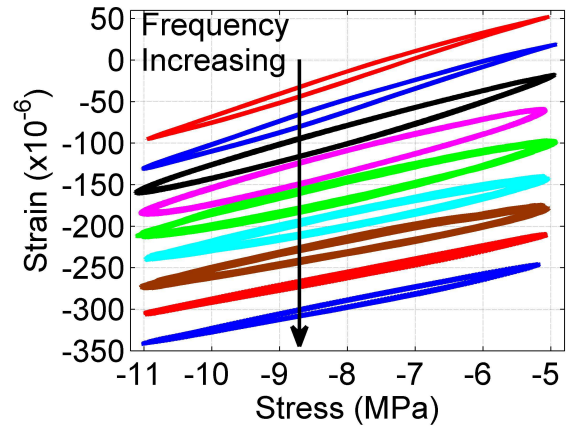

(a)

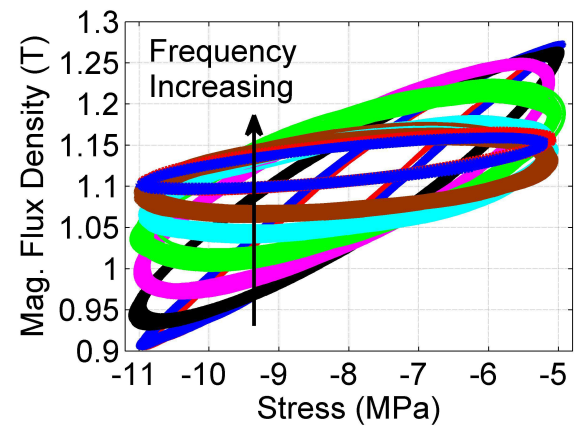

(b)

Figure 9: Minor loop dynamic sensing responses of the solid Galfenol rod for a bias magnetic field of 2.46 $\mathrm{kA} / \mathrm{m}$; strain responses are successively shifted by $-35 \times 10^{-6}$ for visualization purposes.

\section{SUMMARY AND CONCLUSIONS}

This paper summarized the authors' recent report, ${ }^{4}$ in which a detailed and precise characterization of Galfenol's $\left(\mathrm{Fe}_{81.6} \mathrm{Ga}_{18.4}\right)$ magnetic and mechanical responses to dynamic compressive stresses up to $31 \mathrm{MPa}$ and $1 \mathrm{kHz}$ was presented. The objective was to measure the 1D, dynamic sensing response of the material and to quantify from the response, the frequency dependence of the material properties for 1D sensing. This was accomplished by controlling the axial, dynamic stress and static magnetic field over a specific region of a Galfenol rod, and measuring the axial strain and magnetic flux density. Auxiliary variables - drive voltage, drive current, and temperature - were also measured for reference. The design of the experiment was summarized by briefly discussing the specimen design, uncertainty analysis and calibration methods, experimental setup and methods, and data processing methods.

A wide variety of ASTM standards were used to define the specimen's geometric tolerances and to ensure a uniform magnetic excitation of the specimen's gauge region. The estimated uncertainty of most calculated 
quantities met ASTM recommendations. The system was refined to minimize the three key sources of error: (1) electromagnetic noise in strain signals due to Galfenol's magnetic response, (2) error in load signals due to the inertial force of fixturing, and (3) phase misalignment between signals due to conditioning electronics. For dynamic characterization, strain error was kept below $1.2 \%$ of full scale by wiring two collocated gauges in series (noise cancellation) and through lead wire weaving. Inertial force error was kept below $0.41 \%$ by measuring the dynamic force in the specimen using a nearly collocated piezoelectric load washer. The phase response of all conditioning electronics was explicitly measured and corrected for in post processing.

The dynamic sensing responses of the solid Galfenol rod were presented. Current control performed well, but the performance of the field controller significantly degraded above 100 to $200 \mathrm{~Hz}$. In general, as frequency is increased, the sensing response becomes more linear due to an increase in eddy currents, which tend to suppress the active behavior of the material. Hysteresis significantly increases and sensitivity to stress decreases with frequency. As frequency increases above about $100 \mathrm{~Hz}$, the elbow in the strain versus stress response disappears and the region of softened behavior stiffens until it coincides with the saturated, purely-elastic regime. These trends imply that at high frequency, magnetic moment rotation is severely inhibited in the solid Galfenol rod.

This novel dynamic characterization provides a detailed set of data that allows for the validation of recently-developed Galfenol constitutive models that incorporate rate-dependent effects. The frequencydependent material properties (presented in the full report) also permit accurate modeling of Galfenol-based systems that experience time-varying forces.

\section{ACKNOWLEDGMENTS}

This work was supported by the NASA Aeronautics Scholarship Program (grant \# NNX14AE24H). Additional support was provided by the NASA Aeronautics Research Mission Directorate Seedling Fund and the Smart Vehicle Concepts Center (www.SmartVehicleCenter.org), a National Science Foundation Industry/University Cooperative Research Center.

\section{REFERENCES}

[1] Atulasimha, J. and Flatau, A., "A review of magnetostrictive iron-gallium alloys," Smart Materials and Structures 20(4), 043001 (2011).

[2] Walker, T. W., Experimental characterization and modeling of Galfenol (FeGa) alloys for sensing, Master's thesis, The Ohio State University (2012).

[3] Weng, L., Wang, B., Dapino, M., Sun, Y., Wang, L., and Cui, B., "Relationships between magnetization and dynamic stress for Galfenol rod alloy and its application in force sensor," Journal of Applied Physics 113(17), 17 A917 (2013).

[4] Scheidler, J., Asnani, V., and Dapino, M., "Dynamic characterization of Galfenol (Fe81.6 Ga18.4)." NASA Technical Publication (in review).

[5] Weng, L., Walker, T., Deng, Z., Dapino, M., and Wang, B., "Major and minor stress-magnetization loops in textured polycrystalline Fe $81.6 G_{18.4}$ Galfenol," Journal of Applied Physics 113(2), 024508 (2013).

[6] Deng, Z. and Dapino, M. J., "Characterization and finite element modeling of Galfenol minor flux density loops," Journal of Intelligent Material Systems and Structures 26(1), 47-55 (2015).

[7] Figliola, D.E. \& Beasley, R., [Theory and design for mechanical measurements], John Wiley \& Sons, Inc., Hoboken, NJ (2006).

[8] Smith, R. C. and Dapino, M. J., "A homogenized energy framework for ferromagnetic hysteresis," Magnetics, IEEE Transactions on 42(7), 1747-1769 (2006).

[9] ASTM International, "ASTM E 467 - 08: Standard practice for verification of constant amplitude dynamic forces in an axial fatigue testing system," (2008).

[10] ASTM International, "ASTM D5992 - 96: Standard guide for dynamic testing of vulcanized rubber and rubberlike materials using vibratory methods," (2011). 